\title{
Desempeño silvicultural de Plathymenia reticulata con adición de fósforo en el Bosque Atlántico, Brasil
}

\section{Silvicultural performance of Plathymenia reticulata with phosphorous addition in Forest Atlantic, Brazil}

\begin{abstract}
Emanuel França Araújol*, Ray Luiz Babilon Carreço2; Jeferson Pereira Martins Silva², Marília Braz de Carvalho², Kelly Nery Bighi², Elzimar de Oliveira Gonçalves²; Marcos Vinicius Winckler Caldeira² y Tiago de Oliveira Godinho ${ }^{3}$
\end{abstract}

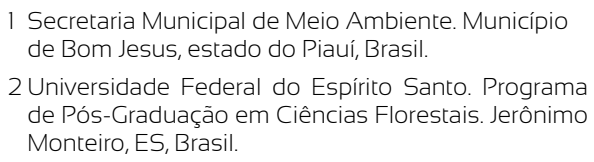

2 Universidade Federal do Espírito Santo. Programa de Pós-Graduação em Ciências Florestais. Jerônimo Monteiro, ES, Brasil.

3 Reserva Natural Vale. Vale S.A. Linhares, ES, Brasil

* Autor de correspondencia. emanuelfa.bj@hotmail.com

\section{RESUMEN}

El objetivo de este estudio fue analizar el crecimiento inicial a mediano plazo de un asentamiento de $P$. reticulata bajo diferentes dosis de fósforo. El experimento se llevó a cabo en un área del Bosque Atlántico, Sooretama, norte del estado de Espírito Santo, Brasil. Se probaron cinco dosis de P por árbol ( $0 \mathrm{~g}, 27 \mathrm{~g}, 47 \mathrm{~g}, 67 \mathrm{~g}$ y $87 \mathrm{~g}$ ) en un diseño de bloques al azar con tres repeticiones y 18 plantas por parcela. A los 3 meses, 9 meses y 15 meses se midió la altura y el diámetro a nivel del suelo. A los 36 meses de la siembra, se midió el diámetro a la altura del pecho, la supervivencia y el área basal y se hizo una evaluación nutricional de los árboles. También se utilizó un modelo logístico para modelar el crecimiento en altura de árboles individuales y el modelo logístico considerando efectos fijos y forma mixta (efectos fijos y aleatorios) para verificar si la inclusión del efecto aleatorio (árbol) resultaría en ganancias en términos de precisión en la estimación de la altura futura de los árboles de $P$. reticulata. La especie presenta potencial para su uso en programas de reforestación en condiciones edafoclimáticas probadas y en condiciones similares. La especie no fue muy exigente en fósforo y la adición de este nutriente promovió efectos negativos en su crecimiento a corto y mediano plazo. Con respecto al modelo de crecimiento en altura, el modelo logístico ajustado en forma mixta mostró ser superior al ajuste tradicional.

PALABRAS CLAVE: leguminosa arbórea, modelos mixtos, nutrición forestal, silvicultura tropical, restauración forestal.

\section{ABSTRACT}

The objective of this study was to analyse the initial medium-term growth of a $P$. reticulata settlement under different phosphorus doses. The experiment was carried out in an area of Atlantic Forest, Sooretama, Northern Espírito Santo State, Brazil. Five doses of P per each tree $(0 \mathrm{~g}, 27 \mathrm{~g}, 47 \mathrm{~g}, 67 \mathrm{~g}$ and $87 \mathrm{~g})$ were tested in a randomized block design with three replicates and 18 plants per plot. At 3 months, 9 months and 15 months, height and diameter were measured at the soil level. At 36 months height, diameter at breast height, survival, basal area, and nutritional evaluation of the trees were measured. A logistic model was also used to model the growth in height of individual trees and a logistic model considering fixed effects and mixed form (fixed and random effects), in order to verify if the inclusion of the random effect (tree) would result in gains in terms of accuracy in the estimation of the future height of $P$. reticulata trees. The species presents potential for use in reforestation programs under edaphoclimatic conditions tested and under similar conditions. The species was not very demanding in $\mathrm{P}$, and the addition of this nutrient promotes negative effects on its growth in the short and medium term. Regarding the model of growth in height, the logistic model adjusted in the mixed form showed to be superior to the traditional adjustment.

KEYWORDS: tree legume, mixed models, forest nutrition, tropical forestry, forest restoration. 


\section{INTRODUCCIÓN}

El suelo brasileño y las condiciones climáticas, en combinación con incentivos fiscales otorgados en la década de 1960, así como la inversión en investigación y tecnología han permitido a la silvicultura nacional brasileña de los géneros Eucalyptus y Pinus alcanzar altos niveles de productividad, convirtiéndose en un modelo de éxito mundial. Recientemente, el interés en la información sobre propagación y desempeño silvícola de especies nativas ha aumentado debido a la necesidad de restaurar o promover la regeneración natural de 18 millones de hectáreas para 2030, meta asumida por Brasil en el el Glasgow en la 26a Conferencia de las Naciones Unidas sobre el Cambio Climático (Ministério do Meio Ambiente, 2021). Los costos de la restauración forestal son altos y para aumentar el éxito de las plantaciones se requiere una base de datos sobre el desempeño de las especies nativas en condiciones de campo bajo diferentes condiciones edafoclimáticas, seleccionando las especies apropiadas (Morais Júnior et al., 2019). Esta base de datos debe incluir los requerimientos para el buen desarrollo de cada especie. En el campo, las plántulas recién plantadas pueden beneficiarse de la fertilización al promover el crecimiento temprano y aumentar las tasas de supervivencia donde la baja fertilidad del suelo y la alta competencia herbácea pueden inhibir el crecimiento (Earnshaw, Baribault y Jacobs, 2016). La dosificación de fertilizantes y la formulación química influyen en su efectividad. El crecimiento inicial de los árboles y la producción de biomasa están más comúnmente limitados por el fósforo (P) (Netzer, Pozzi, Dubbert y Herschbach, 2019), especialmente en suelos degradados en regiones tropicales.

Las especies forestales nativas mostraron una gran variación en el comportamiento de la fertilización con fosfato. Se ha demostrado que las especies pioneras responden a la fertilización y, con el avance del grupo sucesional, el estímulo al crecimiento proporcionado por la fertilización es menos pronunciado y a veces inexistente (Santos, Resende, Neto y Corte, 2008). Se encontraron resultados positivos de la adición de P para Senna macranthera (Fonseca, Paiva y Neves, 2011), Anadenanthera macrocarpa
(Gonçalves, Paiva, Neves y Gomes, 2012), Mimosa caesalpiniaefolia (Gonçalves, Paiva, Lima-Neves y Gomes, 2013), Dalbergia nigra (Gonçalves, Paiva, Neves, Klippel y Caldeira, 2014), Schinopsis brasiliensis (Oliveira, Teixeira-Rios, Melo y Yano-Melo, 2015) y Cassia grandis (Freitas, Paiva, Leite y Oliveira Neto, 2017). Souza et al. (2012) observaron que las plántulas de Peltophorum dubium presentaban altos requerimientos nutricionales para nitrógeno $(\mathrm{N})$, utilizando P de manera más eficiente. Sin embargo, Santos, Resende, Neto y Corte (2008) encontraron que el P tenía poca influencia en el crecimiento y la nutrición de las plántulas de Calophyllum brasiliensis y Myroxylon peruiferum.

La mayoría de los estudios sobre el efecto de la adición de $\mathrm{P}$ en especies forestales nativas se han realizado en condiciones de vivero, descuidando el objetivo principal de la respuesta de campo. Martinotto, Martinotto, Coelho, Azevedo y Figueiredo (2012), evaluando la supervivencia y el crecimiento inicial de seis especies del bosque de Cerrado en consorcio con yuca (Manihot esculenta), encontraron que la fertilización con fosfato favoreció solo a la especie Sclerolobium paniculatum. Por lo tanto, para repetir con especies nativas el éxito alcanzado con especies exóticas, es necesario dominar las etapas de crecimiento y los requisitos de las especies, incluyendo fertilización, encalado, espaciado, manejo y otros.

Vinhático, vinhático-do-campo, candeia e pau-amarelo son algunos nombres populares de Platbymenia reticulata Benth (Fabaceae-Mimosoideae), especie arbórea nativa que crece en una amplia área de distribución natural en el Cerrado, el Bosque Atlántico, Caatinga, Amazon y otros biomas de América del Sur (Carvalho, 2009; Morim, 2015). Descrita como pionera (Morais Junior et al., 2019) o secundaria temprana, esta especie puede presentar individuos adultos de $5 \mathrm{~m}$ a $30 \mathrm{~m}$ de altura con diámetro que varía de $30 \mathrm{~cm}$ a $150 \mathrm{~cm}$, dependiendo del bioma (Carvalho, 2009).

El crecimiento de $P$. reticulata se describe como relativamente rápido, ocurre en suelos de alta y baja fertilidad, tiene potencial para su uso en la recuperación de áreas degradadas, forestación urbana y sector maderero (Lorenzi, 2008; Carvalho, 2009). P. reticulata se usa 
ampliamente en la medicina popular para el tratamiento de infecciones, hemorragias e intoxicaciones por serpientes (Moura et al., 2016). La versatilidad de $P$. reticulata la hace interesante para su uso en programas de restauración forestal y plantaciones comerciales, sin embargo, los trabajos que evalúan su crecimiento en el campo aún son incipientes.

\section{OBJETIVOS}

El objetivo de este estudio fue evaluar el efecto de diferentes dosis de $\mathrm{P}$ en el rendimiento silvícola de $P$. reticulata en un área del Bosque Atlántico en Fazenda São Marcos en Sooretama, norte del estado de Espírito Santo, Brasil. Para esto, se probaron las siguientes hipótesis: (i) $P$. reticulata tiene potencial para su uso en programas de reforestación en el Bosque Atlántico, presentando un rápido crecimiento inicial; (ii) una mayor disponibilidad de $\mathrm{P}$ en el sustrato mejora el crecimiento inicial y a mediano plazo de un rodal monoespecífico de $P$. reticulata.

\section{MATERIALES Y MÉTODOS}

\section{Área de estudio}

El experimento se estableció en un área de 1.31 hectáreas del proyecto Biomas, ubicado en Fazenda São Marcos en Sooretama, norte del estado de Espírito Santo, Brasil, desde abril de 2013 hasta abril de 2016, en las coordenadas $19^{\circ}$ 12 '55 "S y $40^{\circ} 03$ ' 09" W y $43 \mathrm{~m}$ de altitud. El sitio del experimento se inserta en el bioma del Bosque Atlántico con vegetación primaria clasificada como bosque de ombrófilos densos de tierras bajas. El clima de la región es cálido y semihúmedo Aw (Köppen), con una temperatura media anual de $23.5^{\circ} \mathrm{C}$ y una precipitación anual de 1276 mm (Alvares et al., 2013) (Fig. 1).

\section{Efecto de $P$ sobre el rendimiento silvícola de Plathymenia reticulata}

El experimento se realizó en un diseño de bloques al azar con cinco dosis de $\mathrm{P}$ y tres repeticiones/bloques. Los tratamientos consistieron en dosis de 0 g, 27 g, 47 g, $67 \mathrm{~g} \mathrm{y}$ $87 \mathrm{~g}$ de $\mathrm{P}_{2} \mathrm{O}_{5}$ por cada árbol. Cada bloque se subdividió en 5 parcelas de $12 \mathrm{~m} \times 33 \mathrm{~m}\left(396 \mathrm{~m}^{2}\right.$ por parcela). Cada tratamiento consistió en un conjunto de árboles dispuestos en cuatro filas que contenían 11 plantas cada una, sin embargo, para las mediciones se consideró un borde simple, siendo la parcela útil las dos líneas centrales con nueve árboles, con un total de 18 árboles útiles por parcela.

El suelo del área se caracteriza por Argisol amarillo distrófico (Sistema Brasileiro de Classificação de Solos [SiBCS] 2013). Antes de plantar, el área estaba cubierta de pasto con Pennisetum clandestinum. El control de las plantas espontáneas se llevó a cabo aplicando herbicida de glifosato en toda el área del experimento para facilitar las actividades de plantación y realización del experimento. Previo al experimento, se hizo una caracterización de atributos químicos del suelo (Tabla 1), de acuerdo con Donagema, Campos, Calderano, Teixeira y Viana (2011).

La corrección del suelo para la siembra consistió en la aplicación manual de $1.0 \mathrm{Mg} \mathrm{ha}^{-1}$ de piedra caliza dolomítica. Posteriormente, el suelo sebarbechó con un subsoladordescompactador a una profundidad de $60 \mathrm{~cm}$ para facilitar el crecimiento del sistema de raíces de la planta. El espaciamiento entre plántulas adoptado fue de $3 \mathrm{~m} \times 3 \mathrm{~m}$ y para su implantación se excavaron hoyos o pocillos de 30 $\mathrm{cm} \times 30 \mathrm{~cm} \times 30 \mathrm{~cm}$.

Se usó superfosfato simple como fuente de P. Se agregaron al suelo $5 \mathrm{~g}$ de sulfato de zinc por pocillo, junto con la fertilización con P. Las plántulas se compraron en el vivero Vale Natural Reserve en Sooretama y tenían una altura promedio $30 \mathrm{~cm}$. Las plántulas se plantaron tres meses después de la aplicación de dosis de $\mathrm{P}$ en los pozos preexcavados, agregando polímero de hidrocloruro hidratado. Debido a las condiciones climáticas del sitio durante el período de siembra, fue necesario colocar mantillo alrededor de las plántulas para retener la humedad y suavizar la temperatura del suelo y, en consecuencia, la evapotranspiración.

Se aplicaron nitrógeno y potasio en dosis de $20 \mathrm{~g}$ por plántula, de la siguiente manera: $35 \%$ de la dosis total tres meses después de la siembra; $35 \%$ seis meses después de plantar y $30 \%$ un año después de plantar. Además de la primera fertilización de cobertura, también se aplicaron $10 \mathrm{~g}$ de bórax por plántula, por medio de hoyuelos que la rodeaban. 


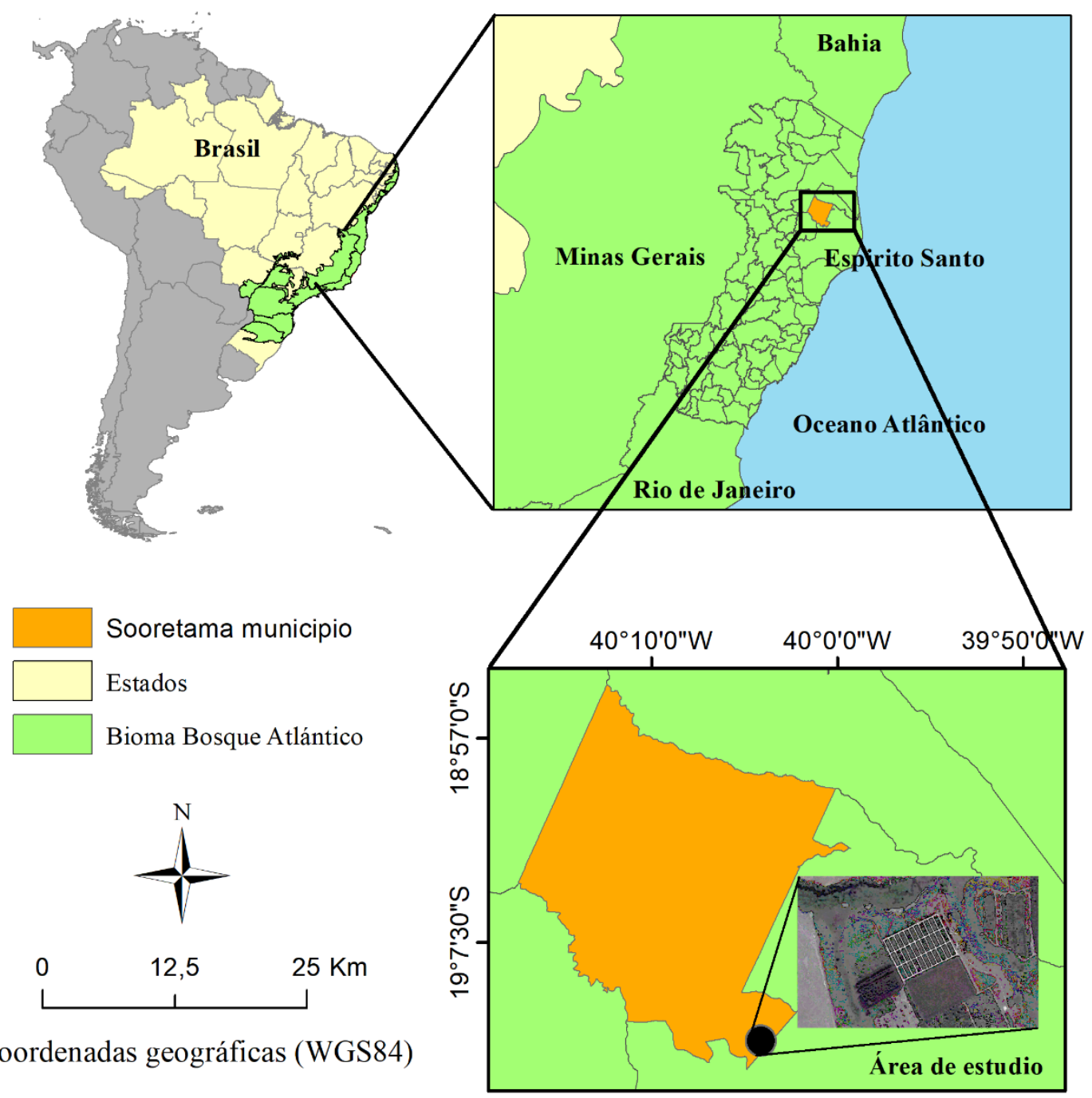

FIGURA 1. Localización del área de estudio.

TABLA 1. Análisis químico del suelo antes de la implantación de Platbymenia reticulata.

\begin{tabular}{|c|c|c|c|c|c|c|c|c|c|c|c|c|}
\hline Prof: & $\mathrm{pH}$ & $P^{*}$ & $K$ & $\begin{array}{c}\mathrm{Ca}+ \\
\mathrm{Mg}\end{array}$ & $A l^{3+}$ & $H+A I$ & $S B$ & $t$ & $T$ & $m$ & V & M.O \\
\hline$(\mathrm{cm})$ & $\mathrm{CaCl}_{2}$ & $\mathrm{mg} \mathrm{dm}^{-3}$ & $\mathrm{mg} \mathrm{dm} \mathrm{m}^{-3}$ & \multicolumn{6}{|c|}{ 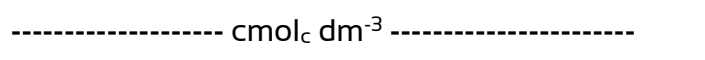 } & \multicolumn{2}{|c|}{---- \% ---- } & $g \mathrm{dm}^{-3}$ \\
\hline $0-12$ & 4.6 & 1.5 & 0.1 & 1.0 & 0.2 & 2.9 & 1.7 & 1.9 & 4.7 & 9.4 & 37.3 & 19.9 \\
\hline $12-30$ & 4.6 & 0.4 & 0.1 & 1.8 & 0.2 & 3.1 & 1.9 & 2.1 & 4.9 & 11.3 & 37.3 & 11.3 \\
\hline
\end{tabular}


A los 3 meses, 9 meses y 15 meses de la siembra, se midieron las siguientes variables: altura total $(\mathrm{H}, \mathrm{m})$ desde el suelo hasta el final del brote apical y diámetro a la altura del suelo (DAS, mm); esta última medición se hizo a una altura de $7 \mathrm{~cm}$. La primera medición de $\mathrm{H}$, a los 3 meses, se realizó con cinta métrica y las dos mediciones posteriores se hicieron con regla telescópica; el DAS se midió con un calibrador digital. A los 36 meses, se midieron la altura total $(\mathrm{H}, \mathrm{cm})$, el diámetro a la altura del pecho (DAP, mm; medido a $1.3 \mathrm{~m}$ sobre el nivel del suelo) con la ayuda de una cinta métrica; el área basal $\left(\mathrm{G}, \mathrm{m}^{2} \mathrm{ha}^{-1}\right)$, mediante la suma de áreas seccionales a $1.3 \mathrm{~m}$, extrapoladas a hectáreas; y supervivencia (\%), contando el número de plántulas vivas en los tiempos de evaluación en función del número de plántulas plantadas.

Para evaluar el estado nutricional de los árboles de $P$. reticulata en diferentes tratamientos, se recolectaron hojas a los 36 meses. La colección se zigzague en la gráfica útil y las hojas se recolectaron en cada punto cardinal en la parte intermedia de la corona. Las muestras de hojas por parcela formaron una muestra compuesta. Las muestras se tomaron para su secado (en un horno de circulación forzada de aire a $60^{\circ} \mathrm{C}$ hasta masa constante), molienda (en el molino Willey) y almacenamiento (en bolsas de papel) y luego se enviaron al laboratorio para análisis químico, de acuerdo con la metodología descrita por Silva (2009).

Se recolectaron muestras simples de hojarasca acumulada, totalizando 10 muestras simples por parcela, en los mismos tiempos y puntos de recolección de hojas. La operación se realizó con la ayuda de una plantilla metálica de $0.25 \mathrm{~m} \times 0.25 \mathrm{~m}$. Después de secar (en un horno de circulación forzada de aire a $60{ }^{\circ} \mathrm{C}$ hasta una masa constante) y medir la masa seca, las 10 muestras de cada parcela se unieron para formar una muestra compuesta. Luego, la muestra fue triturada en un molino tipo Willey, tamizada en una malla de $1.0 \mathrm{~mm}$ de abertura, almacenada en bolsas de papel y enviada para análisis químico (Tabla 2), según Silva (2009). El suelo se caracterizó por atributos químicos al final del experimento a los 36 meses (Tabla 3), de acuerdo con Donagema et al. (2011).

Los datos se sometieron a análisis de regresión para verificar la dosis óptima de P para cada variable, utilizando la primera derivada de los estimadores $\beta_{0}$ y $\beta_{1}$. Para elegir la ecuación, se consideró la importancia de los parámetros, la prueba t, la importancia biológica y el coeficiente de determinación $\left(\mathrm{R}^{2}\right)$. El modelo adoptado fue el lineal $y=\beta_{0}$ $+\beta_{1} x$. Los análisis se realizaron con el programa $\mathrm{R}(\mathrm{R}$ Core Team, 2018) versión 3.5.0.

TABLA 2. Análisis químico de la camada de Plathymenia reticulata en función de la adición de fósforo a los 36 meses.

\begin{tabular}{lcccccc}
\hline $\mathrm{P}_{2} \mathrm{O}_{5}$ & $N$ & $P$ & $K$ & $\mathrm{Ca}$ & $\mathrm{Mg}$ & $S$ \\
\hline $\begin{array}{l}\text { Gramos por } \\
\text { arbol }\end{array}$ & & - & & & \\
0 & 14.96 & 0.39 & 0.98 & 9.13 & 1.50 & 1.53 \\
27 & 15.96 & 0.43 & 1.04 & 9.69 & 1.77 & 1.40 \\
47 & 16.61 & 0.55 & 1.04 & 9.55 & 1.71 & 1.64 \\
67 & 15.56 & 0.53 & 1.59 & 7.11 & 1.54 & 2.09 \\
87 & 12.60 & 0.54 & 1.69 & 6.70 & 1.54 & 1.43 \\
\hline
\end{tabular}


TABLA 3. Análisis químico del suelo a los 36 meses, profundidad $(0$ - 20) cm en la siembra de Plathymenia reticulata.

\begin{tabular}{|c|c|c|c|c|c|c|c|c|c|c|c|c|}
\hline Prof. & $p H$ & $P^{*}$ & $K$ & $\begin{array}{c}\mathrm{Ca}+ \\
\mathrm{Mg}\end{array}$ & $A \beta^{3+}$ & $H+A I$ & $S B$ & $t$ & $T$ & $m$ & V & M.O \\
\hline$(\mathrm{cm})$ & $\mathrm{H}_{2} \mathrm{O}$ & $\mathrm{mg} \mathrm{dm}^{-3}$ & $\mathrm{mg} \mathrm{kg-3}$ & -------. & ------ c & $\mathrm{ll}_{\mathrm{c}} \mathrm{dm}^{-3}$ & 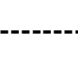 & ---- & & ----- \% ---- & & $\mathrm{g} \mathrm{dm^{-3 }}$ \\
\hline $0-5$ & 6.19 & 2.6 & 74.2 & 2.38 & 0.00 & 1.61 & 2.71 & 2.71 & 4.32 & 0.00 & 62.57 & 2.17 \\
\hline $5-10$ & 5.98 & 2.4 & 63.8 & 1.88 & 0.00 & 1.95 & 2.17 & 2.18 & 4.12 & 0.00 & 52.8 & 2.12 \\
\hline $10-20$ & 5.68 & 2.13 & 52.53 & 1.38 & 0.08 & 2.43 & 1.62 & 1.70 & 4.05 & 5.01 & 39.83 & 2.00 \\
\hline
\end{tabular}

\section{Modelos analizados y estructuras de varianza y autocorrelación}

Se ajustó el modelo logístico comúnmente utilizado para modelar el crecimiento en altura de árboles individuales (Ec. 1).

$$
h=\frac{\beta_{0}}{\left(1+\beta_{1} \cdot e\left(-\beta_{2} \cdot i d\right)\right)} . \varepsilon
$$

Donde:

$\mathrm{h}=\operatorname{altura}(\mathrm{m})$

$i d=$ edad actual (meses)

$\beta_{i}=$ coeficientes del modelo

$\varepsilon=$ error al azar

Como el crecimiento en altura varía de un árbol a otro, el modelo logístico se ajustó para efectos fijos y mixtos (efectos fijos y aleatorios), para ver si la inclusión del efecto aleatorio (árbol) daría lugar a ganancias en términos de precisión en la estimación de la altura futura de los árboles de $P$. reticulata. El modelo logístico se ajustó en la forma mixta de la siguiente manera:

$$
h_{i j}=\frac{\beta_{0}+u_{i l}}{\left(1+\beta_{1} \cdot e\left(-\beta_{2} \cdot i d_{i j}\right)\right)} \cdot \varepsilon_{i j}
$$

Donde:

$h_{i j}=$ altura (m) del i-ésimo árbol en la j-ésima medida

$i d=$ edad del j-ésimo árbol en la i-ésima medida

$\beta_{0}, \beta_{1}, \beta_{2}=$ efectos fijos de los parámetros

$e_{i j}=$ error aleatorio, que se asume que se distribuye como $\mathrm{e}_{\mathrm{ij}} \sim \mathrm{N}(0, \mathrm{Ri})$

$u i=$ efectos aleatorios de los parámetros con una matriz de varianza-covarianza $(\mathrm{D})$, es decir, $u_{i} \sim \mathrm{N}(0, \mathrm{D})$

La matriz de varianza-covarianza (Ri) se puede determinar como:

$$
R i=\sigma^{2} \cdot G_{i}^{0.5} \cdot \Gamma_{i} \cdot G_{i}^{0.5}
$$

Donde:

$\sigma^{2}=$ factor de escala para la dispersión de errores, de modo que $\sigma^{2}$ es igual a la varianza residual del modelo

$G_{i}=$ matriz diagonal para describir la heterocedasticidad del grupo de efectos aleatorios

$\Gamma_{i}=$ matriz de autocorrelación.

En los modelos de crecimiento de árboles individuales, la variación de las alturas generalmente aumenta a medida que los árboles crecen, este efecto se conoce como heterocedasticidad (Pinheiro y Bastes, 2000; Campos y Leite, 2013). En este caso, para corregir dicho efecto, se 
analizaron las funciones varPower (potencia de una covariable) y varIdent (varianza constante por grupo). Además, para corregir una eventual autocorrelación entre los datos observados de medidas repetidas a lo largo del tiempo en el mismo individuo, se probaron dos estructuras de autocorrelación: autorregresiva de primer orden (AR1) y autorregresiva contínua de primer orden (CAR1).

El modelo logístico en la forma fija se ajustó mediante el método Gauss Newton utilizando el software R Core Team (2018), utilizando la función nls. Para ajustar los modelos no lineales mixtos, se utilizó el algoritmo de máxima verosimilitud restringido, disponible en el paquete nlme (Pinheiro y Bates, 2000).

\section{Estimación de la precisión de las estimaciones}

La evaluación de las estimaciones generadas por los modelos mixtos y el modelo fijo se realizó con base en las siguientes estadísticas: sesgo del coeficiente de correlación (r), parcialidad $[\mathrm{P}(\%)]$, raíz de la desviación cuadrática media [RMSE (\%)], criterio de información de Akaike (AIC) y criterio bayesiano (BIC) (Tabla 4). Para complementar las estadísticas, se prepararon gráficos que relacionaban los valores observados y los estimados por los modelos.

\section{Resultados}

A los 3 meses, los resultados mostraron un efecto negativo de las dosis de $\mathrm{P}$ sobre el crecimiento, tanto en $\mathrm{H}$ como en DAS de plántulas de $P$. reticulata. El crecimiento máximo observado, $76.4 \mathrm{~cm}$ en $\mathrm{H}$ y $9.9 \mathrm{~mm}$ en DAS se obtuvo para la dosis de $0 \mathrm{~g}$ de $\mathrm{P}_{2} \mathrm{O}_{5}$ (Fig. 2a, b) y, con cada aumento de dosis de $\mathrm{P}$, se observó una reducción promedio de $6.6 \mathrm{~cm}$ en $\mathrm{H}$ y de $0.9 \mathrm{~mm}$ en DAS.

Se observó la misma tendencia a los 9 meses de crecimiento, el aumento de las dosis de $\mathrm{P}$ redujo linealmente el incremento de $\mathrm{H}$ y DAS de las plántulas de P. reticulata (Fig. 2c, d). Los valores máximos de $\mathrm{H}$ y DAS fueron $2.40 \mathrm{~m}$ y $3.47 \mathrm{~mm}$, respetivamente, observados en la dosis de $0 \mathrm{~g}$ de $\mathrm{P}_{2} \mathrm{O}_{5}$, con una reducción promedio de 31.6 cm y 3.8 mm en cada aumento de dosis de P.
A los 15 meses de crecimiento, el soporte de $P$. reticulata todavía tuvo un efecto negativo de la fertilización con fosfato, la mayor altura observada fue $4.16 \mathrm{~m}$, se observó para la dosis de $0 \mathrm{~g}$ de $\mathrm{P}_{2} \mathrm{O}_{5}$, con una tasa de crecimiento de reducción en el orden de $20.4 \mathrm{~cm}$ por cada aumento de dosis de P (Fig. 2e). También se encontró que a los 15 meses de crecimiento las diferencias presentadas para DAS en función de la adición de $\mathrm{P}$ tendían a desaparecer, presentando un promedio general de $5.42 \mathrm{~cm}$ (Fig. 2f).

Con respecto a los 36 meses de crecimiento, no hubo efecto de la adición de $\mathrm{P}$ sobre el crecimiento del árbol de $P$. reticulata, el rodal presentó un promedio de $\mathrm{H}$ de $5.9 \mathrm{~m}$ y DAP de $9.51 \mathrm{~cm}$ (Fig. 3a, b). Sin embargo, se encontró que la fertilización con fosfato promovió una reducción lineal significativa en la supervivencia de los árboles, lo que refleja la reducción del área basal. Se observaron los mejores resultados para la supervivencia $(92.92 \%)$ y el área basal (7.34 $\mathrm{m}^{2} \mathrm{ha}^{-1}$ ) para la dosis de $0 \mathrm{~g}$ de $\mathrm{P}_{2} \mathrm{O}_{5}$ (Fig. 3c, d).

No se observó ningún efecto de la adición de $\mathrm{P}$ en la plantación sobre la nutrición de los árboles de $P$. reticulata a los 36 meses de crecimiento, excepto por el contenido de P de la hoja, que mostró una tendencia de crecimiento lineal creciente con el mejor resultado siendo $0.82 \mathrm{~g} \mathrm{~kg}^{-1}$, a $87 \mathrm{~g}$ de P2O5 (Fig. 4b). En general, los árboles de P. reticulata presentaron el siguiente orden de absorción $\mathrm{N}>\mathrm{Ca}>\mathrm{K}>$ $\mathrm{Mg}>\mathrm{S}>\mathrm{P}$.

Los parámetros estimados del modelo logístico de efectos fijos para la estimación de altura futura, así como los errores estándar respectivos y las pruebas de significación se pueden observar en la tabla 5. Se puede observar que todos los parámetros del modelo fueron significativos $(\mathrm{p}<0.01)$ por la prueba $\mathrm{t}$.

La tabla 6 muestra los indicadores estadísticos utilizados para evaluar la calidad de ajuste de los modelos de efectos mixtos y su forma original. Los ajustes de modelos mixtos con diferentes estructuras de varianzacovarianza y autocorrelación dieron como resultado estimaciones RMSE (\%) que van desde 20.58 a 36. Se observó sesgo y los coeficientes de correlación fueron mayores a 0.88 . 

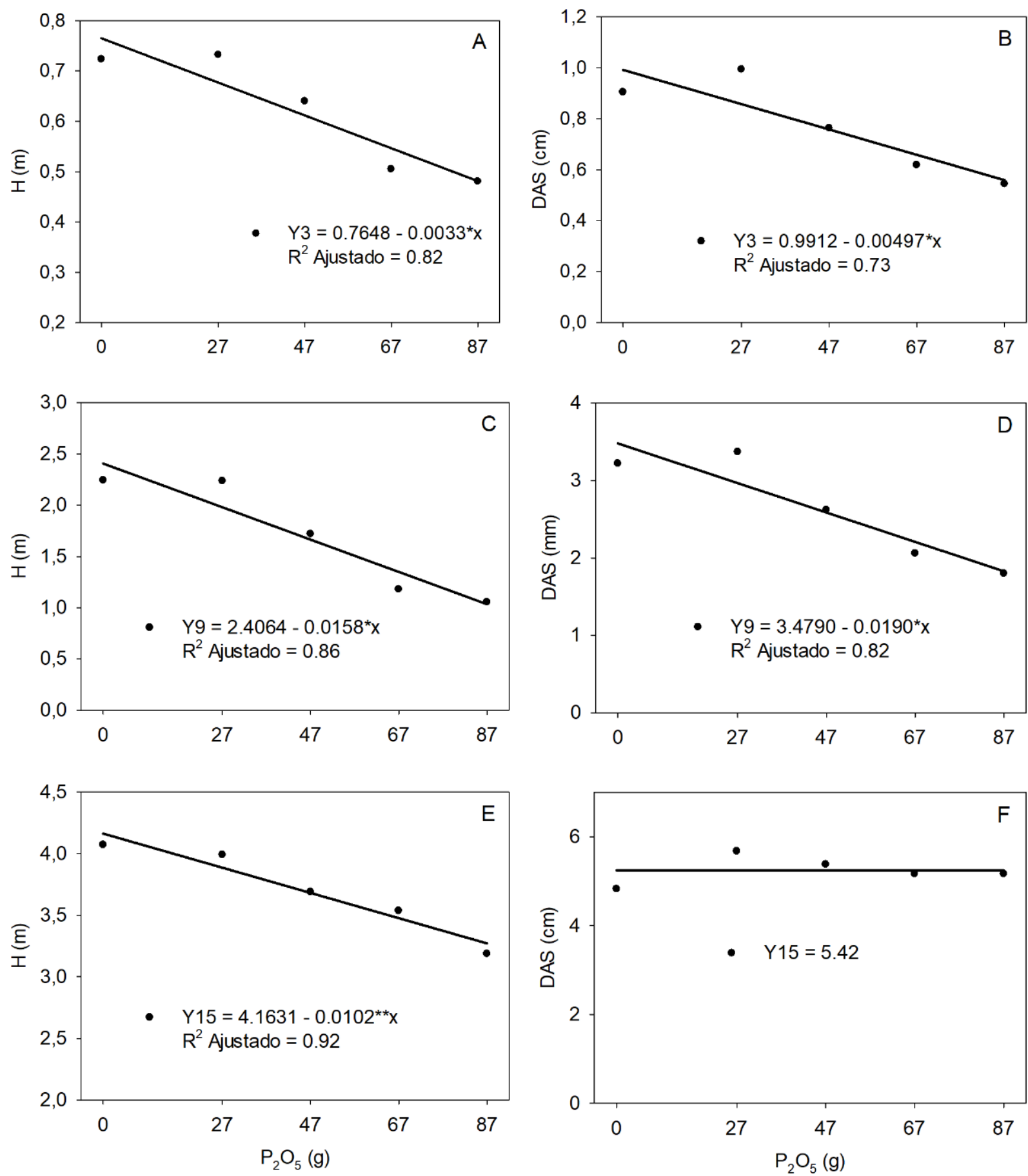

Figura 2. Valores dasométricos de plántulas de Platbymenia reticulata en función de la adición de fósforo. (A) Altura a los 3 meses de crecimiento; (B) diámetro a $7 \mathrm{~cm}$ de nivel del suelo a los 3 meses; (C) altura a los 9 meses; (D) diámetro a $7 \mathrm{~cm}$ de nivel del suelo a los 9 meses; (E) altura a los 15 meses; (F) diámetro a $7 \mathrm{~cm}$ de nivel del suelo a los 15 meses. ${ }^{*} \mathrm{y}{ }^{* *}$ significativo al nivel de $5 \%$ y $1 \%$ de probabilidad por la prueba $\mathrm{F}$ respectivamente ns no significativo a $5 \%$ de probabilidad, por la prueba t. 

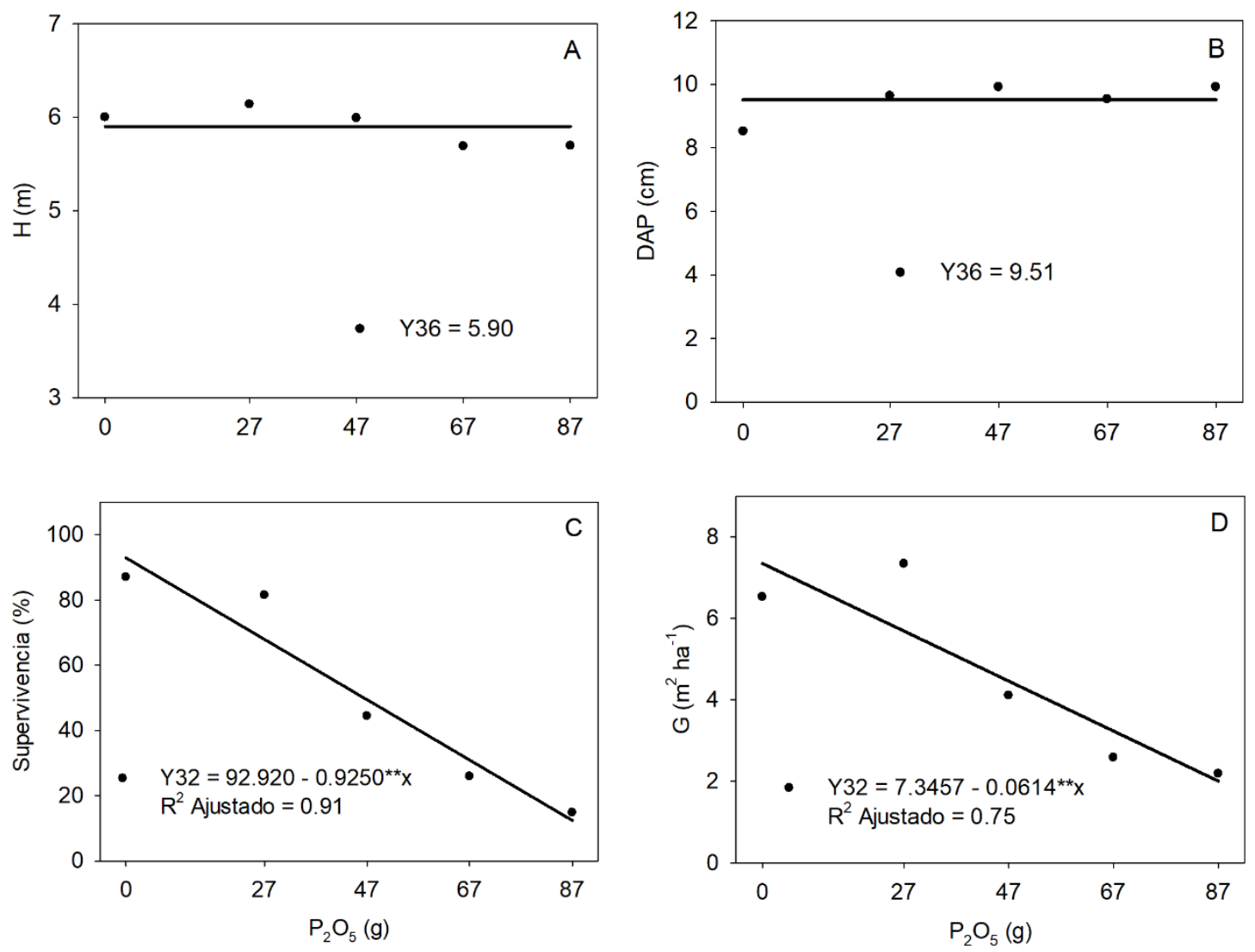

Figura 3. Valores dasométricos de árboles de Plathymenia reticulata a los 36 meses debido a la adición de fósforo. (A) altura; (B) diámetro a la altura del pecho; (C) supervivencia; (D) área basal.

* ${ }^{* *}$ significativo al nivel del $5 \%$ y $1 \%$ de probabilidad por la prueba $F$ respectivamente

ns no significativo al 5\% de probabilidad, por la prueba t.

En general, los modelos mixtos (modelos 2 y 3 ) obtuvieron una mayor precisión en el ajuste y la homogeneidad de los valores estadísticos en comparación con el modelo logístico en su forma fija (Modelo 1). Sin embargo, los modelos 4 y 5 dieron como resultado valores más discrepantes en comparación con otros modelos, considerando la RMSE (\%). Con respecto a las estadísticas de las estimaciones que utilizan los modelos mixtos, los ajustes que utilizan la función varldent fueron superiores en comparación con la función varPower.

Al observar los gráficos de la relación entre la altura observada y la estimada (Fig. 4), es evidente que el modelo logístico ajustado solo con efecto fijo (Fig. 5b) presentó una mayor dispersión en relación con la línea ideal (1:1), que representa menor precisión. En el modelo mixto ajustado
(Fig. 5a), los puntos fueron más agregados y uniformes, lo que corrobora los resultados de los indicadores estadísticos que se muestran en la tabla 6 .

\section{DISCUSIÓN}

En general, se confirma la primera hipótesis planteada, $P$. reticulata mostró una alta supervivencia y un rápido crecimiento inicial, lo que indica que la especie tiene un alto potencial para su uso en programas de reforestación en el Bosque Atlántico. También hubo una tendencia de mejora en los atributos químicos del suelo después de 36 meses de asentamiento, con énfasis en los aumentos de $\mathrm{P}, \mathrm{Ca}+\mathrm{Mg}$, suma base, CTC efectivo, saturación de bases y materia orgánica (Tabla 3), indicando el comienzo de los procesos de ciclo de nutrientes. 

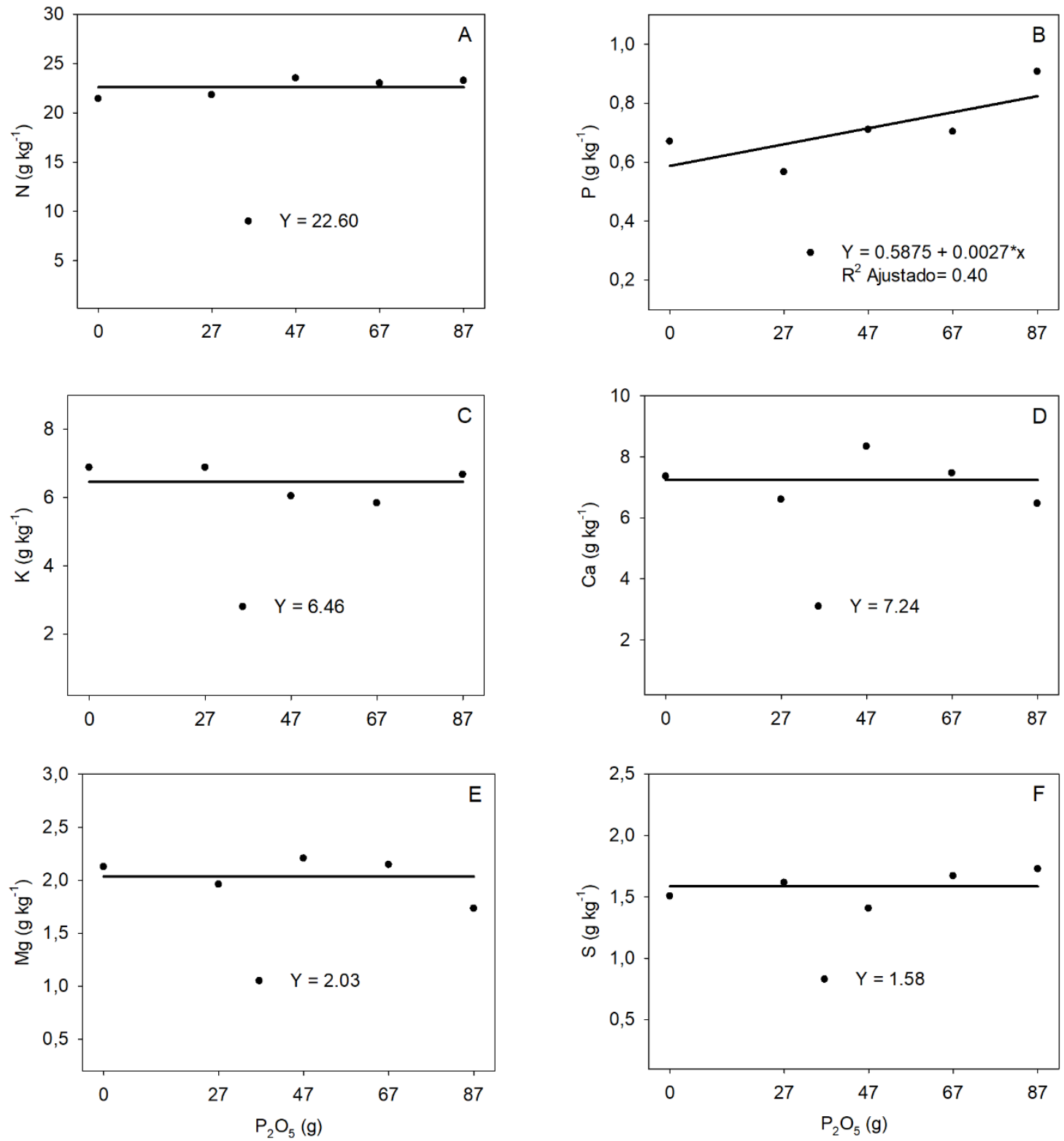

Figura 4. Contenido de nutrientes en las hojas de los árboles de Platbymenia reticulata a los 36 meses de crecimiento en función de la adición de fósforo. (A) nitrógeno; (B) fósforo; (C) potasio; (D) calcio; (E) magnesio; (F) azufre.

* $y$ ** significativo al nivel de $5 \%$ y $1 \%$ de probabilidad por la prueba $F$, respectivamente. ns no significativo al 5\% de probabilidad, por la prueba t.

TABLA 5. Estadísticas de ajuste de los modelos en su forma original para estimar la altura futura de los árboles de Plathymenia reticulata.

\begin{tabular}{cccc} 
Estimar & Error estándar & tc & Pr $>\mid t c l$ \\
\hline 6.48689 & 0.86446 & 7.504 & $<0.001$ \\
21.5194 & 2.11995 & 10.151 & $<0.001$ \\
0.23403 & 0.02098 & 11.154 & $<0.001$ \\
\hline
\end{tabular}


TABLA 6. Indicadores estadísticos utilizados para evaluar la calidad de ajuste de los modelos de efectos mixtos y su forma original en Plathymenia reticulata.

\begin{tabular}{cccccccc}
\hline Modelo & F. variación & F. autocorrelación & RMSE (\%) & $P(\%)$ & $r$ & AIC & BIC \\
\hline 1 & - & - & 34.86 & 0.380 & 0.88 & 1012.08 & 1028.82 \\
2 & varldent & AR1 & 20.58 & -0.26 & 0.96 & 868.47 & 893.54 \\
3 & varldent & CARl & 20.07 & -0.24 & 0.96 & 874.61 & 912.2118 \\
4 & varPower & AR1 & 36.02 & -6.05 & 0.88 & 1074.61 & 1012.2118 \\
5 & varPower & CAR1 & 36.02 & -6.05 & 0.88 & 1074.61 & 1012.2118 \\
\hline
\end{tabular}

Modelo 1

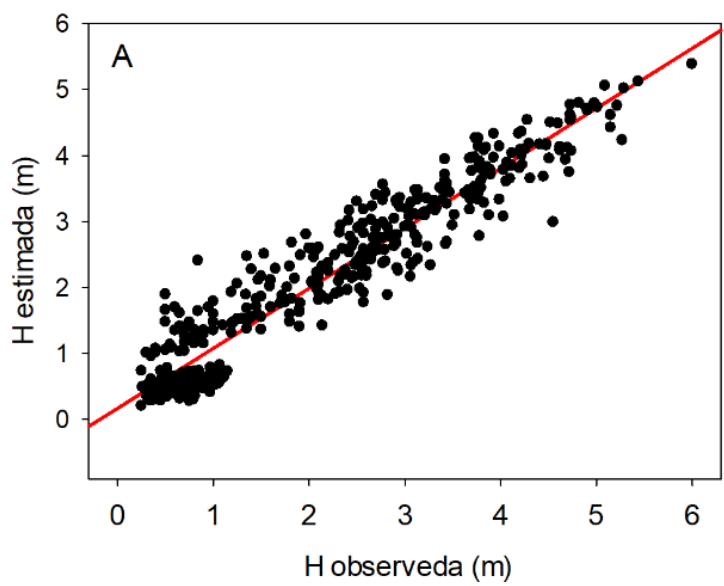

FIGURA 5. Gráficos de la relación entre altura estimada y observada para evaluar el crecimiento en altura de los árboles de Plathymenia reticulata.
Modelo 2

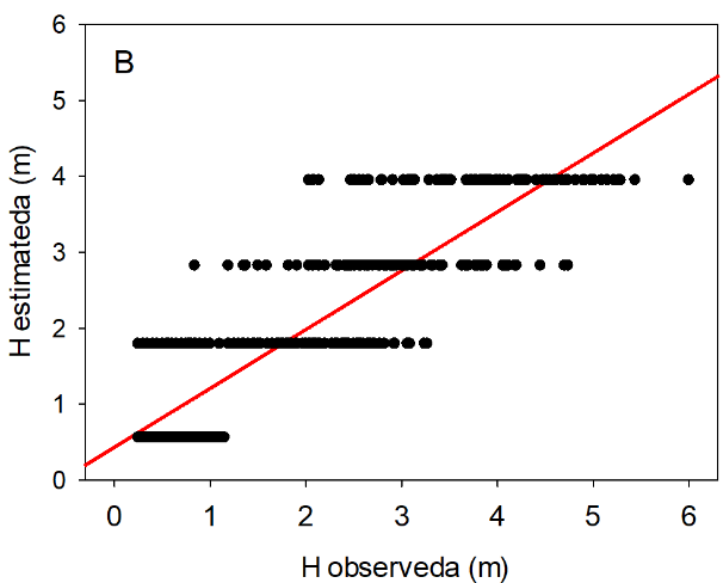

Hay pocas plantaciones de la especie a nivel experimental, comercial y de recomposición, lo que dificulta una comparación más segura. Morais Junior et al. (2019), con el objetivo de identificar el potencial de 25 especies de árboles que compensan el carbono en un área degradada del Bosque Atlántico, en el estado de Minas Gerais, descubrieron que Plathymenia foliolosa Benth (sinónimo de $P$. reticulata) tenía un alto potencial para tales plantaciones debido a su alta tasa de supervivencia $(75 \%)$, su crecimiento inicial en $\mathrm{H}(5.34 \mathrm{~m})$, DAS $(13.43 \mathrm{~cm})$ y su incremento promedio de carbono $(29.1 \mathrm{~kg}$ año-1) a los 29 meses. Los resultados de crecimiento existentes de $P$. reticulata son similares a los obtenidos en este estudio.

Sin embargo, el rendimiento silvícola de $P$. reticulata fue influenciado negativamente por la adición de $\mathrm{P}$, lo que contradice la segunda hipótesis, ya que las especies de leguminosas de la sucesión ecológica temprana generalmente presentan una alta demanda nutricional. Aunque las diferencias en $\mathrm{H}$ y DAS fueron más prominentes en los primeros meses de implantación y desaparecieron a los 36 meses, el aumento en la fertilización con fosfato promovió una reducción significativa en el número de individuos sobrevivientes, lo que refleja la disminución en el área basal baja.

Millones de años de evolución han ayudado a las plantas a desarrollar una serie de mecanismos genéticos, morfológicos, fisiológicos y bioquímicos de adaptación a los hábitats pobres de P. Estos mecanismos dan como resultado una mayor adquisición de $\mathrm{P}$ y un uso interno más eficiente (Rao, Miles, Beebe y Horst, 2016) e incluyen la 
modificación de la arquitectura de la raíz, el desarrollo de un sistema radical extenso, raíces más largas y raíces más delgadas, acidificación de rizosfera, exudación de ácidos orgánicos, expresión de transportadores de $\mathrm{P}$ de alta afinidad, eficiencia de $\mathrm{P}$ de la homeostasis citoplasmática y removilización interna de $\mathrm{P}$, secreción de fosfatasa $\mathrm{y}$ simbiosis micorrízica (Makoi, Chimphango y Dakora, 2010; Balemi y Negisho, 2012; Folli-Pereira, Meira-Haddad, Bazzolli y Kasuya, 2012). Por lo tanto, se sugiere que $P$. reticulata tiene una alta adaptabilidad a las condiciones restrictivas de $\mathrm{P}$, ya que originalmente el suelo tenía en promedio $0.73 \mathrm{mg} \mathrm{dm}^{-3}$ de P en las capas analizadas (Tabla 1), un valor que se considera muy bajo. Los niveles de $\mathrm{P}$ de las hojas en los árboles a menudo se usan como un indicador del suministro de P (Fäth, Kohlpaintner, Göttlein y Mellert, 2019), sin embargo, la sutil tendencia al alza en las hojas de $P$. reticulata encontradas a los 36 meses en función del aumento de la fertilización con fosfato no fue acompañada por ningún efecto positivo del crecimiento del asentamiento. Esto es aún más evidente cuando se observan los niveles de P que se encuentran en la camada (Tabla 2), que presentó el más bajo entre los macronutrientes, lo que indica que la especie tiene una baja demanda o alta eficiencia de utilización de este nutriente.

Silva et al. (2006), evaluando la eficiencia en el uso de nutrientes por tres especies de árboles pioneros que se encuentran con frecuencia en pasturas degradadas donde el $\mathrm{P}$ siempre es limitante, después de encalado y la aplicación de $\mathrm{P}$, verificaron diferencias entre las especies estudiadas. Vismia japurensis utilizó la mayoría de los nutrientes analizados de manera más eficiente, especialmente $\mathrm{N}, \mathrm{P}$ y $\mathrm{Mg}$, con altos valores de tasa fotosintética, bajos contenidos foliares de $\mathrm{P}$, y la limitación de estos nutrientes no fue limitante para el buen desempeño de esta especie, como es el caso de $P$. reticulata en este estudio. Por lo tanto, a pesar de los notables avances en la silvicultura nativa, se necesitan estudios para establecer condiciones nutricionales óptimas para cada especie cultivada en suelos con baja disponibilidad nutricional.

Con respecto al modelo de crecimiento en altura para la especie estudiada en este trabajo, es evidente la ventaja del modelo logístico ajustado en forma mixta en comparación con el ajuste tradicional. Esto se debe al modelo mixto propuesto que presenta el árbol como un componente aleatorio. En este caso, considerar el árbol en el análisis significa asignar curvas de crecimiento a cada uno de los árboles estudiados, reduciendo así el error cometido. Es razonable decir que este es el factor principal para reducir la RMSE de 34.86\% (modelo 1) a 20.07\% (modelo 3).

Una de las ventajas de los modelos que incluyen efectos aleatorios en su estructura en comparación con los modelos de efectos fijos es la reducción del error estándar residual. Obras como Mendonça, Pádua Chaves y Calegario (2015) obtuvieron una ganancia de precisión de $53.89 \%$ en modelos hipsométricos; Carvalho et al. (2011), tuvieron una reducción en el error estándar residual (Syx) de 26.31\% a $4.01 \%$ en predicción volumétrica. Gouveia, Silva, Ferreira, Gadelha y Lima-Filho (2015), también trabajando con modelos volumétricos, obtuvieron una reducción en el error estándar residual de 26 veces.

Los modelos de regresión tradicionales requieren supuestos de cumplimiento como la independencia entre las observaciones y la homogeneidad de la varianza, que no siempre se satisfacen (Gouveia et al., 2015). Una alternativa a estos modelos es la sustitución por modelos mixtos, que permiten generalizar las estructuras de correlación espaciotemporal y la varianza no constante, extrayendo mejor las relaciones de variables independientes con datos agrupados en comparación con los modelos de regresión tradicionales (Fausto, Carneiro, Antunes, Pinto y Colosimo, 2008; Gouveia et al., 2015). Esto demuestra una vez más la ventaja de este tipo de modelos aplicados a los datos con medidas repetidas en el tiempo para evaluar el crecimiento de las especies forestales, como se estudia en esta investigación.

\section{CONCLUSIONES}

La especie Plathymenia reticulata tiene potencial para su uso en programas de reforestación bajo condiciones edafoclimáticas probadas y condiciones similares.

La especie no es exigente en $\mathrm{P}$, la adición de este nutriente promueve efectos negativos en su crecimiento a corto y mediano plazos. 
Con respecto al modelado de crecimiento en altura, el modelo logístico ajustado en la forma mixta fue superior al tradicional.

\section{RECONOCIMIENTOS}

Este trabajo se realizó con el apoyo de Coordenação de Aperfeiçoamento de Pessoal de Nivel Superior - Brasil (CAPES) y de la Fundação de Amparo à Pesquisa e Inovação do Espirito Santo (FAPES).

\section{REFERENCIAS}

Alvares, C. A., Stape, J. L., Sentelhas, P. C., de Moraes, G., Leonardo, J., \& Sparovek, G. (2013). Köppen's climate classification map for Brazil. Meteorologische Zeitschrift, 22(6), 711-728. doi: 10.1127/09412948/2013/0507

Balemi, T., \& Negisho, K. (2012). Management of soil phosphorus and plant adaptation mechanisms to phosphorus stress for sustainable crop production: a review. Journal of soil science and plant nutrition, 12(3), 547-562. doi: 10.4067/S0718-95162012005000015

Campos, J. C. C. \& Leite, H. G. (2013). Mensuração florestal: perguntas e respostas. atual. e ampl. Viçosa-MG: UFV, Imprensa Universitária.

Carvalho, P. E. R. (2009) Espécies arbóreas brasileiras. Colombo; Brasília: Embrapa Informação Tecnológica, 2009. v. 3.

Carvalho, S. P. C., Calegario, N., Fonseca, F., Borges, L. A. C., de Mendonça, A. R., \& de Lima, M. P. (2011). Modelos não lineares generalizados aplicados na predição da área basal e volume de Eucalyptus clonal. Cerne, 17(4), 541-548. doi: 10.1590/S010477602011000400013

Donagema, G. K.; Campos, D. B.; Calderano, S. B.; Teixeira, W. G.; Viana, J. M. (2011). Manual de métodos de análise de solos. (p. 225). Rio de Janeiro: Embrapa Solos.

Earnshaw, K. M., Baribault, T. W., \& Jacobs, D. F. (2016). Alternative field fertilization techniques to promote restoration of leguminous Acacia koa on contrasting tropical sites. Forest Ecology and Management, 376, 126-134. doi: 10.1016/j.foreco.2016.06.012

Fäth, J., Kohlpaintner, M., Blum, U., Göttlein, A., \& Mellert, K. H. (2019). Assessing phosphorus nutrition of the main European tree species by simple soil extraction methods. Forest ecology and management, 432, 895-901. doi: 10.1016/j.foreco.2018.10.007

Fausto, M. A., Carneiro, M., Antunes, C. M. D. F., Pinto, J. A., \& Colosimo, E. A. (2008). O modelo de regressão linear misto para dados longitudinais: uma aplicação na análise de dados antropométricos desbalanceados. Cadernos de Saúde Pública, 24, 513-524. doi: 10.1590/S0102-311X2008000300005
Folli-Pereira, M. S., Meira-Haddad, L. S. A., Bazzolli, D. M. S., \& Kasuya, M. C. M. (2012). Micorriza arbuscular e a tolerância das plantas ao estresse. Revista Brasileira de Ciência do Solo, 36(6), 1663-1679. doi: 10.1590/S0100-06832012000600001

Fonseca, C. A., de Paiva, H. N., \& Neves, J. C. L. (2011). Resposta de mudas de Senna macranthera cultivadas em Argissolo VermelhoAmarelo a macronutrientes. Ciência Florestal, 21(1), 63-76. doi: $10.5902 / 198050982748$

Freitas, E. C. S., de Paiva, H. N., Leite, H. G., \& de Oliveira Neto, S. N. (2017). Crescimento e qualidade de mudas de Cassia grandis linnaeus f. em resposta à adubação fosfatada e calagem. Ciência Florestal, 27(2), 509-519. doi:10.5902/1980509827732

Gonçalves, E. O., de Paiva, H. N., Neves, J. C. L., \& Gomes, J. M. (2012). Nutrição de mudas de angico-vermelho (Anadenanthera macrocarpa (Benth.) Brenan) submetidas a doses de N, P, K, Ca e Mg. Revista Árvore, 36(2), 219-228. doi: 10.1590/S0100-67622012000200003

Gonçalves, E. O., de Paiva, H. N., de Lima Neves, J. C., \& Gomes, J. M. (2013). Nutrição de mudas de Mimosa caesalpiniaefolia Benth. sob diferentes doses de N, P, K, Ca e Mg. Ciência Florestal, 23(2), 273286. doi:10.5902/198050989274

Gonçalves, E. O.; de Paiva, H. N., Neves, J. C. L., Klippel, V. H., \& Caldeira, M. V. W. (2014). Crescimento de jacarandá-da-bahia (Dalbergia nigra ((Vell.) Fr. All. ex Benth)) sob diferentes doses de NPK. Cerne, 20(3), 493-500. doi: 10.1590/01047760201420031220

Gouveia, J, F.; da Silva, J. A. A., Ferreira, R. L. C., Gadelha, F. H. L., \& Lima-Filho, L. M. A (2015). Modelos volumétricos mistos em clones de Eucalyptus no Polo Gesseiro do Araripe, Pernambuco. Floresta, 45(3), 587-598. doi: 10.5380/rf.v45i3.36844

Lorenzi, H.; Souza, V. C. (2008) Botânica Sistemática (2a ed.). São Paulo: Nova Odessa.

Makoi, J. H., Chimphango, S. B., \& Dakora, F. D. (2010). Elevated levels of acid and alkaline phosphatase activity in roots and rhizosphere of cowpea (Vigna unguiculata L. Walp.) genotypes grown in mixed culture and at different densities with sorghum (Sorghum bicolor L.). Crop and Pasture Science, 61(4), 279-286. doi: 10.1071/CP09212

Martinotto, F., Martinotto, C., Coelho, M. D. F. B., de Azevedo, R. A. B., \& de Figueiredo, M. C. (2012). Sobrevivência e crescimento inicial de espécies arbóreas nativas do Cerrado em consórcio com mandioca. Pesquisa Agropecuária Brasileira, 47(1), 22-29. doi: 10.1590/S0100-204X2012000100004

Mendonça, A. R., de Pádua Chaves, S., \& Calegario, N. (2015). Modelos hipsométricos generalizados mistos na predição da altura de 
Eucalyptus sp. Cerne, 21(1), 107-115. doi: 10.1590/01047760201521011191

Ministério do Meio Ambiente (2021). Com meta ambiciosa, Brasil anuncia redução de 50\% nas emissões de carbono até 2030. Brasil. Recuperado de https://www.gov.br/mma/pt$\mathrm{br} /$ noticias/anuncio-de-ampliacao-da-meta-de-reducao-deemissoes-para-50-ate-2030-e-destaque-nas-primeiras-

participacoes-do-brasil-na-cop26

Morais Junior, V. T. M., Jacovine, L. A. G., Torres, C. M. M. E., Alves, E. B. B. M., de Paiva, H. N., Alcántara-de la Cruz, R., \& Zanuncio, J. C. (2019). Early assessment of tree species with potential for carbon offset plantations in degraded area from the southeastern Brazil. Ecological indicators, 98, 854-860. doi: 10.1016/j.ecolind.2018.12.004

Morim, M. P. (2015) Plathymenia in lista de espécies da flora do Brasil. Jardim Botânico do Rio de Janeiro. Recuperado de http://reflora.jbrj.gov.br/jabot/floradobrasil/FB83636

Moura, V. M., da Silva, W. C. R., Raposo, J. D., Freitas-de-Sousa, L. A., Dos-Santos, M. C., de Oliveira, R. B., \& Mourão, R. H. V. (2016). The inhibitory potential of the condensed-tannin-rich fraction of Plathymenia reticulata Benth. (Fabaceae) against Bothrops atrox envenomation. Journal of ethnopharmacology, 183, 136-142.

Netzer, F., Pozzi, L., Dubbert, D., \& Herschbach, C. (2019). Improved photosynthesis and growth of poplar during nitrogen fertilization is accompanied by phosphorus depletion that indicates phosphorus remobilization from older stem tissues. Environmental and Experimental Botany, 162, 421-432. doi: 10.1016/j.envexpbot.2019.03.017

Oliveira, J. R. G. D., Teixeira-Rios, T., Melo, N. F. D., \& Yano-Melo, A. M. (2015). Response of an endangered tree species from Caatinga to mycorrhization and phosphorus fertilization. Acta Botanica Brasilica, 29(1), 94-102. doi: 10.1590/0102-33062014abb3420

Pinheiro, J. C. \& bates D. M. (2000). Mixed-effects models in S and S-PLUS: New York Springer-Verlag.

R Development Core Team (2018). R: a language and environment for statistical computing. Vienna, Austria: R Foundation for Statistical Computing. https://www.R-project.org/.

Rao, I. M., Miles, J. W., Beebe, S. E., \& Horst, W. J. (2016). Root adaptations to soils with low fertility and aluminium toxicity. Annals of Botany, 118(4), 593-605. doi: 10.1093/aob/mcw073.

Santos, J. Z. L., de Resende, Á. V., Neto, A. E. F., \& Corte, E. F. (2008). Crescimento, acúmulo de fósforo e frações fosfatadas em mudas de sete espécies arbóreas nativas. Revista Árvore, 32(5), 799-807. doi: 10.1590/S0100-67622008000500003.

SIBCS, Sistema Brasileiro de Classificação de Solos. 3. ed. Rio de Janeiro: Empresa Brasileira de Pesquisa Agropecuária. Centro Nacional de Pesquisa em Solos. 2013. 353p.

Silva, C. E. M., Carvalho Gonçalves, J. F., Luizão, F. J., Feldpausch, T. R., Silva, C. E. M., Morais, R. R (2006). Eficiência no uso dos nutrientes por espécies pioneiras crescidas em pastagens degradadas na Amazônia central. Acta Amazonica, 36(4), 503-512. doi: 10.1590/S0044-59672006000400011

Silva, F. C. D. A. S. (2009). Manual de análises químicas de solos, plantas e fertilizantes. 2. ed. (p. 627). Brasília, DF: Embrapa Informação Tecnológica.

Souza, N. H., Marchetti, M. E., de Oliveira Carnevali, T., Ramos, D. D., Scalon, S. D. P. Q., \& da Silva, E. F. (2012). Estudo nutricional da canafístula (II): eficiência nutricional em função da adubação com nitrogênio e fósforo. Revista Árvore, 36(5), 803-812. doi: 10.1590/S0100-67622012000500002.

Manuscrito recibido el 17 de septiembre de 2019

Aceptado el 20 de octubre de 2020

Publicado el 13 de diciembre de 2021

Este documento se debe citar como:

Araújo, E. F., Carreço, L. R. B., Silva, J. P. M., Carvalho, M. B., Bighi, K. N., Gonçalves, E. O., Caldeira, M. V. W., \& Godinho, T. O. (2021). Desempeño silvicultural de Plathymenia reticulata con adición de fósforo en el Bosque Atlántico Atlántico, Brasil. Madera y Bosques, 27(2), e2722042. doi: 10.21829/myb.2021.2722042

Madera y Bosques por Instituto de Ecología, A.C. se distribuye bajo una Licencia Creative Commons Atribución-NoComercialCompartirlgual 4.0 Internacional. 